\title{
Homomorphisms from a finite groups into wreath products
}

\author{
Jan-Christoph Schlage-Puchta
}

\begin{abstract}
Let $G$ be a finite group, $A$ a finite abelian group. Each homomorphism $\varphi: G \rightarrow A \curlywedge S_{n}$ induces a homomorphism $\bar{\varphi}: G \rightarrow A$ in a natural way. We show that as $\varphi$ is chosen randomly, then the distribution of $\bar{\varphi}$ is close to uniform. As application we prove a conjecture of T. Müller on the number of homomorphisms from a finite group into Weyl groups of type $D_{n}$.
\end{abstract}

Mathematics Subject Classification (2010). 20P05, 20E22.

Keywords. Wreath products, Homomorphism numbers, Weyl groups.

Let $G$ be a finite group, $A$ a finite abelian group. In this article we consider the number of homomorphisms $G \rightarrow A<S_{n}$, where $n$ tends to infinity. These numbers are of interest, since they encode information on the isomorphism types of subgroups of index $n$, confer [2], [3]. If $\varphi: G \rightarrow A<S_{n}$ is a homomorphism, we can construct a homomorphism $\bar{\varphi}: G \rightarrow A$ as follows. We represent the element $\varphi(g) \in A$ 々 $S_{n}$ as $\left(\pi ; a_{1}, \ldots, a_{n}\right)$, where $\pi \in A_{n}$ and $a_{i} \in A$, and then define $\bar{\varphi}(g)=\prod_{i=1}^{n} a_{i}$. The fact that $\bar{\varphi}$ is a homomorphism follows from the fact that $A$ is abelian and the definition of the product within a wreath product. In this article we prove the following.

Theorem 1. Let $G$ be a finite group of order $d, A$ a finite abelian group. Define the distribution function $\delta_{n}$ on $\operatorname{Hom}(G, A)$ as the image of the uniform distribution on $\operatorname{Hom}\left(G, A \backslash S_{n}\right)$ under the map $\varphi \mapsto \bar{\varphi}$. Then there exist positive constants $c, C$, independent of $n$, such that $\left\|\delta_{n}-u\right\|_{\infty}<C e^{-c n^{1 / d}}$, where $u$ is the uniform distribution, and $\|\cdot\|_{\infty}$ denotes the supremum norm.

As an application we prove the following, which confirms a conjecture by T. Müller.

Corollary 2. For a finite group $G$ there exists a constant $c>0$, such that if $W_{n}$ denotes the Weyl group of type $D_{n}$, then

$$
\left|\operatorname{Hom}\left(G, W_{n}\right)\right|=\left(\frac{1}{1+s_{2}(G)}+\mathcal{O}\left(e^{-c n^{1 / d}}\right)\right)\left|\operatorname{Hom}\left(G, C_{2} \succ S_{n}\right)\right|
$$


This assertion was proven by T. Müller under the assumption that $G$ is cyclic (confer [1, Proposition 3]). Different from his approach we do not enumerate homomorphisms $\varphi$ with given image $\bar{\varphi}$, but directly work with the distribution of $\bar{\varphi}$, that is, we obtain the relation between $\left|\operatorname{Hom}\left(G, W_{n}\right)\right|$ and $\left|\operatorname{Hom}\left(G, C_{2} \prec S_{n}\right)\right|$ without actually computing these functions.

Denote by $\pi: A<S_{n} \rightarrow S_{n}$ the canonical projection onto the active group. The idea of the proof is to stratisfy the set $\left|\operatorname{Hom}\left(G, A \zeta S_{n}\right)\right|$ according to $\pi \circ \varphi \in \operatorname{Hom}\left(G, S_{n}\right)$. It turns out that in strata such that $\pi \circ \varphi(G)$ viewed as a permutation group on $\{1, \ldots, n\}$ has a fixed point the distribution of $\bar{\varphi}$ is actually uniform, while the probability of having no fixed point is very small.

Lemma 3. Let $\varphi_{1}: G \rightarrow S_{n}$ be a homomorphism. Let $\varphi: G \rightarrow A \backslash S_{n}$ be a homomorphism chosen at random with respect to the constraint $\pi \circ \varphi=\varphi_{1}$. If the action of $G$ on $\{1, \ldots, n\}$ induced by $\varphi_{1}$ has a fixed point, then $\bar{\varphi}$ is uniformly distributed on $\operatorname{Hom}(G, A)$.

Proof. Without loss we may assume that the point 1 is fixed. A homomorphism $\varphi: G \rightarrow A$ 々 $S_{n}$ with $\pi \circ \varphi=\varphi_{1}$ can be viewed as a pair of homomorphisms $\psi_{1}: G \rightarrow A$ 々 $S_{1}, \psi_{2}: G \rightarrow A<S_{n-1}$, where the projection of $\psi_{2}$ to $S_{n-1}$ is given by the restriction of $\varphi_{1}$ to $\{2, \ldots, n\}$. If we chose $\varphi$ at random, then $\psi_{1}$ and $\psi_{2}$ are independent, and $\psi_{1}$ is clearly uniformly distributed. Now $\bar{\varphi}$ is the sum of two independent random elements in $\operatorname{Hom}(G, A)$, one of which is uniformly distributed. Since $\operatorname{Hom}(G, A)$ with pointwise addition is an abelian group, we conclude that $\bar{\varphi}$ is uniformly distributed.

To bound the number of homomorphisms $\varphi$ for which $\pi \circ \varphi$ has no fixed point we need the following, which is contained in [2, Proposition 1], in particular the equality of equations (8) and (9) in that article.

Lemma 4. Let $G$ be a group, $A$ a finite abelian group, $U$ a subgroup of index $k, \varphi_{1}: G \rightarrow S_{k}$ the permutation representation given by the action of $G$ on $G / U$. Then the number of homomorphisms $\varphi: G \rightarrow A \curlywedge S_{k}$ with $\pi \circ \varphi=\varphi_{1}$ equals $|A|^{k-1}|\operatorname{Hom}(U, A)|$.

We use this to prove the following.

Lemma 5. Let $G$ be a group of order d, $A$ a finite abelian group, $\varphi: G \rightarrow A 2 S_{n}$ be a homomorphism chosen at random. Then there is a constant $c>0$, depending only on $G$, such that the probability that $\pi \circ \varphi(G)$ has no fixed points is $\mathcal{O}\left(e^{-c n^{1 / d}}\right)$.

Proof. Let $U_{1}, \ldots, U_{\ell}$ be a complete list of subgroups of $G$, where $U_{\ell}=G$. To determine a homomorphism $\varphi: G \rightarrow A$ \& $S_{n}$ we first have to chose a homomorphism $\varphi_{1}: G \rightarrow S_{n}$, and then count the number of ways in which this homomorphism can be extended to a homomorphism to $A$ < $S_{n}$. Suppose that the action of $G$ on $\{1, \ldots, n\}$ induced by $\varphi_{1}$ has $m_{i}$ orbits on which $G$ acts similar to the action of $G$ on $G / U$. Then by the previous lemma we find 
that there are

$$
\prod_{i=1}^{\ell}\left(|A|^{\left(G: U_{i}\right)-1}\left|\operatorname{Hom}\left(U_{i}, A\right)\right|\right)^{m_{i}}
$$

possibilities to extend $\varphi_{1}$. The number of ways to choose $\varphi_{1}$ given $m_{1}, \ldots, m_{\ell}$ is $\frac{n !}{\prod_{i=1}^{\ell} m_{i} !\left(G: U_{\ell}\right)^{m_{i}}}$, hence, we obtain

$$
\left|\operatorname{Hom}\left(G, A \curlywedge S_{n}\right)\right|=n ! \sum_{\substack{m_{1}, \ldots, m_{\ell} \\ m_{1}+\cdots+m_{\ell}=n}} \prod_{i=1}^{\ell} \frac{1}{m_{i} !}\left(\frac{|A|^{\left(G: U_{i}\right)-1}\left|\operatorname{Hom}\left(U_{i}, A\right)\right|}{\left(G: U_{i}\right)}\right)^{m_{i}} .
$$

We claim that terms with $m_{\ell}=0$ are small when compared to the whole sum. Since the number of summands is polynomial in $n$, it suffices to show that for every tuple $\left(m_{1}, \ldots, m_{\ell-1}, 0\right)$ there exists a tuple $\left(m_{1}^{\prime}, \ldots, m_{\ell-1}^{\prime}, m_{\ell}^{\prime}\right)$ with $m_{\ell}^{\prime} \neq 0$, such that the summand corresponding to the first tuple is smaller than the one corresponding to the second by a factor $e^{c n^{1 / d}}$. We do so by explicitly constructing the second tuple. Without loss we may assume that $m_{1}$ is maximal in the first tuple. We then set $m_{1}^{\prime}=m_{1}-\left\lfloor c n^{1 / d}\right\rfloor$, $m_{\ell}^{\prime}=\left(G: U_{1}\right)\left\lfloor c n^{1 / d}\right\rfloor$, and $m_{i}^{\prime}=m_{i}$ for $i \neq 1, \ell$, where $c$ is a positive constant chosen later. Then the product on the right hand side of the last displayed equation changes by a factor

$$
\frac{m_{1} !}{\left(m_{1}-\left\lfloor c n^{1 / d}\right\rfloor\right) !}\left(\frac{|A|^{\left(G: U_{1}\right)-1}\left|\operatorname{Hom}\left(U_{1}, A\right)\right|}{\left(G: U_{1}\right)|\operatorname{Hom}(G, A)|^{\left(G: U_{1}\right)}}\right)^{\left.-\left\lfloor c n^{1 / d}\right\rfloor\right)} \frac{1}{\left(\left(G: U_{1}\right)\left\lfloor c n^{1 / d}\right\rfloor\right) !} .
$$

We may assume that $n$ is sufficiently large, so that $m_{1}>2\left\lfloor c n^{1 / d}\right\rfloor$. We can then estimate the factorials using the largest and smallest factors occurring. The other terms can be bounded rather careless to find that this quotient is at least

$$
\left(\frac{m_{1}}{\left(c d n^{1 / d}|A|\right)^{d}\left|\operatorname{Hom}\left(U_{1}, A\right)\right|}\right)^{\left\lfloor c n^{1 / d}\right\rfloor} .
$$

Since $m_{1}$ was chosen maximal we have $m_{1} \geq \frac{n}{|G| \ell}$, and we find that for $c^{-1}=e d|A|\left(\ell \mid \operatorname{Hom}\left(U_{1}, A\right)\right)$ that the last expression is at least $e^{\left\lfloor c n^{1 / d}\right\rfloor}$. Since $c$ depends only on the subgroup $U_{1}$, we can take the minimum value over all the finitely many subgroups and obtain that there exists an absolute constant $c>0$, such that the number of homomorphisms $\varphi$ such that $\pi \circ \varphi$ has no fixed point is by factor $\mathcal{O}\left(e^{-c n^{1 / d}}\right)$ smaller than the number of all homomorphisms.

The theorem now follows from Lemma 1 and 3 .

To deduce the corollary note that $W_{n}$ is the subgroup of $C_{2} 2 S_{n}$ defined by the condition $\left(\pi ; a_{1}, \ldots, a_{n}\right) \in W_{n} \Leftrightarrow a_{1}+\cdots+a_{n}=0$, that is, a homomorphism $\varphi: G \rightarrow C_{2}$ 乙 $S_{n}$ has image in $W_{n}$ if and only if $\bar{\varphi}: G \rightarrow C_{2}$ is trivial. By the theorem the probability for this event differs from the probability that a random homomorphism $G \rightarrow C_{2}$ is trivial by $\mathcal{O}\left(e^{-c n^{1 / d}}\right)$, hence, 
we have

$$
\left|\operatorname{Hom}\left(G, W_{n}\right)\right|=\left(\frac{1}{\left|\operatorname{Hom}\left(G, C_{2}\right)\right|}+\mathcal{O}\left(e^{-c n^{1 / d}}\right)\right)\left|\operatorname{Hom}\left(G, C_{2} \imath S_{n}\right)\right| .
$$

But there is a bijection between non-trivial homomorphisms $G \rightarrow C_{2}$ and subgroups of index 2 , hence, $\left|\operatorname{Hom}\left(G, C_{2}\right)\right|=1+s_{2}(G)$, and the corollary follows.

\section{References}

[1] T. Müller, Enumerating representations in finite wreath products, Adv. in Math. 153 (2000), 118-154.

[2] T. Müller, J.-C. Schlage-Puchta, Classification and Statistics of Finite Index Subgroups in Free Products, Adv. Math. 188 (2004), 1-50.

[3] T. Müller, J.-C. Schlage-Puchta, Statistics of Isomorphism types in free products, Advances in Math. 224 (2010), 707-720.

Jan-Christoph Schlage-Puchta

Krijgslaan 281

Gebouw S22

9000 Gent

Belgium

e-mail: jcsp@cage.ugent.be 